\title{
CLASSICAL ANALOGS FOR QUANTUM SYSTEMS
}

\author{
Nicola CUFARO PETRONI \\ Dipartimento di Fisica dell'Università di Bari e INFN, Sezione di Bari, Bari, Italy
}

Received 1 August 1987; accepted for publication 14 August 1987

Communicated by J.P. Vigier

\begin{abstract}
It is shown that suitable classical analogs for the relativistic quantum systems of two particles always exist, and that they are destitute of causal anomalies.
\end{abstract}

The wave mechanics lives in a configuration space; the classical, hamiltonian mechanics in a phase space. This is but one of the difficulties faced by all the attempts of building a coherent interpretation of the quantum phenomena in terms of classical concepts. The prescription of the right extension procedures from one formulation to another will play, in fact, an essential role in the comprehension of the dynamics of the classical analogs. Moreover, from the beginning of its history, the causal interpretation of the quantum mechanics for two or more particles is tied to the problem of the direct interactions among them [1]. If this poses no serious challenges in a non-relativistic theory, in a relativistic one we must verify that such a causal description is in fact possible. In this letter we will try to formulate suitable classical analogs for the relativistic quantum mechanics of two particles and verify their internal consistency as a preliminary, essential step for all the future developments.

We will start our dicussion with a short and rather intuitive outline of the covariant, canonical formalism describing the dynamics of two point-like, classical, interacting particles of identical mass $m$ [2]. Let us consider a set of canonical variables $q_{a}^{\mu}, p_{a}^{\mu}$ $(a=1,2 ; \mu=0,1,2,3)$ for our two-particle system. The evolution in terms of two scalar parameters $\tau_{a}$, in general not coincident with the usual proper times, is given by means of phase space abstract orbits (surfaces) $q_{a}^{\mu}\left(\tau_{1}, \tau_{2}\right), p_{a}^{\mu}\left(\tau_{1}, \tau_{2}\right)$. This evolution is ruled by the canonical equations $\frac{\partial q_{a}^{\mu}}{\partial \tau_{b}}=\left\{q^{\mu}, H_{b}\right\}, \quad \frac{\partial p_{a}^{\mu}}{\partial \tau_{b}}=\left\{p_{a}^{\mu}, H_{b}\right\}$,

written in terms of scalar hamiltonians of the form

$H_{a}\left(q_{1}^{\mu}, q_{2}^{\mu} ; p_{1}^{\mu}, p_{2}^{\mu}\right)=\frac{1}{2 m} p_{a}^{\mu} p_{a \mu}+V_{a}\left(q_{1}^{\mu}, q_{2}^{\mu} ; p_{1}^{\mu}, p_{2}^{\mu}\right)$,

where, in general, $V_{a}$ contain direct interactions between the two particles. These scalar hamiltonians, describing the evolution in $\tau_{a}$, are related to the mass $m$ and must not be confused with the energy. An equivalent, but less symmetric, description of the evolution in $q_{a}^{0}$ is indeed possible by means of nonscalar hamiltonians related to the energy. The Cauchy problem of our system consists in finding a unique phase space orbit obeying (1), for given initial data. We remark here that we do not impose constraints on our canonical momenta: the $p_{a}^{2}$ are not a priori coincident with $m^{2}$. The reasons will be clear later. It is also well known that our dynamical problem has an equivalent formulation in terms of a system of Hamilton-Jacobi equations of the form

$H_{a}\left(q_{1}^{\mu}, q_{2}^{\mu} ; \partial W / \partial q_{1}^{\mu}, \partial W / \partial q_{2}^{\mu}\right)=\frac{1}{2} m$.

A complete integral of (3) $W\left(q_{1}^{\mu}, q_{2}^{\mu} ; P_{1}^{\mu}, P_{2}^{\mu}\right)$, parametrized by means of the constants $P_{a}^{\mu}$, will play the role of the generating function of the canonical transformation

$p_{a}^{\mu}=\partial W / \partial q_{a \mu}, \quad Q_{a}^{\mu}=\partial W / \partial P_{a \mu}$, 
which trivialize the movement [3].

The endeavour of finding orbits in a relativistic, canonical formalism in presence of an action-at-adistance, must not be considered in contradiction with the no-interaction theorem [4]. In fact, when a direct interaction is present, we can solve our Cauchy problem in the phase space (if the compatibility conditions discussed later are satisfied), but we can no longer interpret the $q_{a}^{\mu}$ as the true positions in the spacetime, since these cannot be canonical variables. Indeed the $q_{a}^{\mu}$ can be interpreted as positions only when they satisfy the relations

$\partial q_{1}^{\mu} / \partial \tau_{2}=\partial q_{2}^{\mu} / \partial \tau_{1}=0$,

or even, from (1),

$\left\{q_{1}^{\mu}, H_{2}\right\}=\left\{q_{2}^{\mu}, H_{1}\right\}=0$,

expressing the fact that eventually, if we want to define true world lines from the abstract phase space orbits, each particle position must depend only on its own time parameter. However, it can be proved that, if the canonical variables $q_{a}^{\mu}$ of our hamiltonian, Poincaré-invariant system satisfy also the relations (6), then the accelerations must vanish and the system must be considered as non-interacting [2]. Hence we will be obliged to solve our Cauchy problem in the phase space and then deduce the world lines in the ordinary spacetime by somehow determining the true, uncanonical positions

$x_{a}^{\mu}\left(q_{1}^{\mu}, q_{2}^{\mu} ; p_{1}^{\mu}, p_{2}^{\mu}\right)$

among the phase space functions satisfying the position equations

$\left\{x_{1}^{\mu}, H_{2}\right\}=\left\{x_{2}^{\mu}, H_{1}\right\}=0$.

The compatibility of the Cauchy problem for the canonical equations (1) deserves a last remark. The evolution of an arbitrary phase-space function $f\left(q_{1}^{\mu}, q_{2}^{\mu} ; p_{1}^{\mu}, p_{2}^{\mu}\right)$ is ruled by $\# 1$

$\partial f / \partial \tau_{a}=\left\{f, H_{a}\right\}$.

It is very easy to see that, if we use $(8)$ in the obvious relation

\footnotetext{
\# We adopted the symbol of partial derivative since $f$ depends on two parameters, $\tau_{1}$ and $\tau_{2}$, through its dependence on $q_{a}^{\mu}$ and $p_{a}^{\mu}$ : it does not indicate any explicit dependence of $f$ on $\tau_{\text {, }}$ and $\tau_{2}$.
}

$\partial^{2} f / \partial \tau_{1} \partial \tau_{2}=\partial^{2} f / \partial \tau_{2} \partial \tau_{1}$

the Jacobi identity and the arbitrariness of $f$ imply

$$
\left\{H_{1}, H_{2}\right\}=0 \text {, }
$$

or even, from eq. (2),

$\left\{V_{1}, V_{2}\right\}+p_{1}^{\mu} \partial V_{2} / \partial q_{1}^{\mu}-p_{2}^{\mu} \partial V_{1} / \partial q_{2}^{\mu}=0$.

The Frobenius theorem [5] states that $(10)$ is even a requirement sufficient to ensure the existence of the orbits $q_{a}^{\mu}\left(\tau_{1}, \tau_{2}\right), p_{a}^{\mu}\left(\tau_{1}, \tau_{2}\right)$ in a unique way, assuming given initial conditions.

We pass now to discuss if a quantum system of two free particles can be described by means of this classical formalism. By quantizing in the usual way the hamiltonians of two free, classical particles

$H_{\mathrm{ca}}=(1 / 2 m) p_{a}^{\mu} p_{a \mu}$

we get the following system of two wave equations $(\hbar=c=1)$ :

$\left(\square_{a}+m^{2}\right) \psi\left(q_{1}^{\mu}, q_{2}^{\mu}\right)=0$.

The following ansatz on an arbitrary, but fixed, solution of (13)

$\psi_{0}\left(q_{1}^{\mu}, q_{2}^{\mu}\right)=R_{0}\left(q_{1}^{\mu}, q_{2}^{\mu}\right) \exp \left[\mathrm{i} W_{0}\left(q_{1}^{\mu}, q_{2}^{\mu}\right)\right]$

allows now a separation of the real and the imaginary parts of (13), so that we have the equivalent system

$\frac{1}{2 m} \frac{\partial W_{0}}{\partial q_{a}^{\mu}} \frac{\partial W_{0}}{\partial q_{a \mu}}-\frac{1}{2 m} \frac{\square_{a} R_{0}}{R_{0}}=\frac{1}{2} m$,

$\frac{\partial}{\partial q_{a}^{\mu}}\left(R_{0}^{2} \frac{\partial W_{0}}{\partial q_{a \mu}}\right)=0$.

Despite an obvious analogy, the usual identification [1] of (15) with a Hamilton-Jacobi equation deserves a short discussion. The Hamilton-Jacobi equations of a classical system of two particles, with potentials $-\square_{a} R_{0} / 2 m R_{0}$, are

$\frac{1}{2 m} \frac{\partial W}{\partial q_{a}^{\mu}} \frac{\partial W}{\partial q_{a \mu}}-\frac{1}{2 m} \frac{\square_{a} R_{0}}{R_{0}}=\frac{1}{2} m$,

where we dropped the label " 0 " in $W$ in order to make clear that now $W$ is a generic solution of (17) for a fixed $R_{0}$, and not the phase $W_{0}$ of $\psi_{0}$, which is only one particular solution of (17). The form of (17) means that, for a fixed $\psi_{0}$, we are considering the 
classical system ruled by the following hamiltonians,

$H_{0 a}=\frac{1}{2 m} p_{a}^{\mu} p_{a \mu}-\frac{1}{2 m} \frac{\square_{a} R_{0}}{R_{0}}$.

However, for a fixed $\psi_{0}$, only the solution $W=W_{0}$ will correspond to a quantum state solution of (13). All the other solutions $W$, relative to the same $R_{0}$, are not, in general, phases of a wavefunction with amplitude $R_{0}$. Of course these remarks hold for each particular wavefunction $\psi_{0}$.

By summarizing: we can say that the wave equations (13) are not connected to only one system of equations (17), but to an entire set of systems (17) with the different potentials derivable from all the possible $R_{0}$ of (14). Moreover, for each Hamilton-Jacobi equation (17), the wave equations (13) select only one solution, $W=W_{0}$, as a possible phase of a wavefunction. That means also that we cannot find the complete integral $W\left(q_{1}^{\mu}, q_{2}^{\mu} ; P_{1}^{\mu}, P_{2}^{\mu}\right)$ needed in the Hamilton-Jacobi theory by looking only at the solutions $W_{0}$ derivable from a quantum mechanical wavefunction. That is why, in the usual causal interpretation of the quantum mechanics [1], we have only the relation

$p_{a}^{\mu}=\partial W_{0} / \partial q_{a \mu}$,

which represents just a half of the canonical transformation (4). In other words, the fact that $W_{0}$ is the unique solution of (17) susceptible of an interpretation as phase of a wavefunction indicates that a quantum system in $\psi_{0}$ must be considered as the restriction, to the phase space surface $\Sigma_{0}$ defined by (19), of the classical system utilized for its description.

At this point we need to introduce the following definition: we will say that a quantum system of two free particles in the state $\psi_{0}$ admits a suitable classical analog when we can find two hamiltonian functions $H_{a}\left(q_{1}^{\mu}, q_{2}^{\mu} ; p_{1}^{\mu}, p_{2}^{\mu}\right)$, satisfying the compatibility conditions (10), leading to time-like trajectories in the ordinary sapcetime and such that their restrictions on $\Sigma_{0}$ satisfy the following relations,

$$
\begin{aligned}
& \left.H_{a}\right|_{\Sigma_{0}}=H_{a}\left(q_{1}^{\mu}, q_{2}^{\mu} ; \frac{\partial W_{0}}{\partial q_{1 \mu}}, \frac{\partial W_{0}}{\partial q_{2 \mu}}\right) \\
& =\frac{1}{2 m} \frac{\partial W_{0}}{\partial q_{a}^{\mu}} \frac{\partial W_{0}}{\partial q_{a \mu}}-\frac{1}{2 m} \frac{\square_{a} R_{0}}{R_{0}}=\frac{1}{2} m,
\end{aligned}
$$

namely such that $W_{0}\left(q_{1}^{\mu}, q_{2}^{\mu}\right)$ is a particular solution of the corresponding Hamilton-Jacobi system. It is clear that the form of $H_{a}$ will depend on the particular, fixed wavefunction $\psi_{0}$. However it should be remarked that in general the requirements listed above may not fix the classical analog in a unique way.

In the light of this definition it is clear now that $H_{0 a}$ of eq. (18) does not give a suitable classical ana$\log$ for our quantum system. In fact, while its restriction on $\Sigma_{0}$ verifies the relations (20), the compatibility conditions (10) are satisfied only by a too restrictive number of states. Indeed the form (18) of $H_{0 a}$, which is the simplest extension of (15) to the entire phase space, indicates that we have chosen as classical extended potentials the so-called quantum potentials

$V_{0 a}\left(q_{1}^{\mu}, q_{2}^{\mu}\right)=-\frac{1}{2 m} \frac{\square_{a} R_{0}}{R_{0}}$,

which are independent of the canonical momenta $p_{a}^{\mu}$. However, if $V_{0 a}$ depends only on $q_{a}^{\mu}$, we have $\left\{V_{01}, V_{02}\right\}=0$, so that, from the arbitrariness of $p_{a}^{\mu}$ (which are independent, unconstrained variables) the compatibility relations in the form (11) imply

$\partial V_{01} / \partial q_{2}^{\mu}=\partial V_{02} / \partial q_{1}^{\mu}=0$.

It means that $V_{01}\left(V_{02}\right)$ cannot depend on $q_{2}^{\mu}\left(q_{1}^{\mu}\right)$ and hence that we can describe only systems with zero direct interaction. This is a too restrictive requirement, not satisfied by every potential (21) derived from a non-factorizable wavefunction $\psi_{0}$. It also clarifies the difficulties of the previous approaches to this problem, based on the naive extension (18) of our dynamics to the entire phase space [6]. Moreover, even in the case of a single, relativistic, quantum particle, it can be shown that the naive extension from $\Sigma_{0}$ to the entire phase space

$H_{0}=\frac{1}{2 m} p^{\mu} p_{\mu}-\frac{1}{2 m} \frac{\square R_{0}}{R_{0}}$

leads to the following relation,

$\dot{q}_{\mu}=\mathrm{d} q_{\mu} / \mathrm{d} \tau=\left\{q_{\mu}, H_{0}\right\}=m^{-1} p_{\mu}$,

and hence on $\Sigma_{0}$ we will have

$\left.\dot{q}_{\mu}\right|_{\Sigma_{0}}=m^{-1} \partial W_{0} / \partial q^{\mu}$. 
But, as can be shown by means of simple examples [7], this implies that these velocities $\left.\dot{q}_{\mu}(\tau)\right|_{\Sigma_{0}}$ can be both time-like and space-like, so that they violate an essential requirement of the idea of causality.

In order to show that a suitable classical analog of our two-body quantum system does in fact exist, we propose here an alternative extension based on the remark that all the hamiltonians of the form

$H_{a}=\frac{1}{2 m}\left(p_{a}^{\prime \prime}-\frac{\partial A}{\partial q_{a \mu}}\right)\left(p_{a \mu}-\frac{\partial A}{\partial q_{a}^{\mu}}\right)$,

where $A\left(q_{1}^{\mu}, q_{2}^{\mu}\right)$ is an arbitrary scalar function of the canonical coordinates only, always satisfy the compatibility conditions (10). For these hamiltonians we have

$\frac{\partial q_{a}^{\mu}}{\partial \tau_{b}}=\left\{q_{a}^{\mu}, H_{h}\right\}=\delta_{a h} \frac{1}{m}\left(p_{a}^{\mu}-\frac{\partial A}{\partial q_{a \mu}}\right)$,

so that $q_{a}^{\mu}$ verify the position equations (7) and are good candidates to play the role of the true positions: indeed (26) indicates that $q_{a}^{\mu}\left(\tau_{a}\right)$ depends only on its own time parameter, so that it defines a true world line. Another consequence of $(26)$ is that the velocities $\partial q_{a}^{\mu} / \partial \tau_{a}$ are not parallel to $p_{a}^{\mu}$, differently from the case of the naive extension (18). Moreover, it can be verified that

$\frac{\partial^{2} q_{a}^{\mu}}{\partial \tau_{a}^{2}}=\left\{\left\{q_{a}^{\mu}, H_{a}\right\}, H_{a}\right\}=0$,

so that the trajectories in the configuration space are straight lines. Hence, if they are time-like somewhere in the configuration space, they will retain this character along all their evolution.

We must now define $A$ so that (26) will take the correct value (20) when restricted on $\Sigma_{0}$. We propose, as the simplest choice,

$A\left(q_{1}^{\mu}, q_{2}^{\mu}\right)=W_{0}\left(q_{1}^{\mu}, q_{2}^{\mu}\right)-W_{\mathrm{F}}\left(q_{1}^{\mu}, q_{2}^{\mu}\right)$,

where $W_{0}$ is defined in (14) and $W_{\mathrm{F}}$ satisfies

$\frac{1}{2 m} \frac{\partial W_{\mathrm{F}}}{\partial q_{a}^{\mu}} \frac{\partial W_{\mathrm{F}}}{\partial q_{a \mu}}=\frac{1}{2} m$.

It is easy to see that the corresponding hamiltonians

$$
\begin{gathered}
H_{\mathrm{F} a}=\frac{1}{2 m}\left(p_{a}^{\mu}-\frac{\partial\left(W_{0}-W_{\mathrm{F}}\right)}{\partial q_{a \mu}}\right)\left(p_{a \mu}-\frac{\partial\left(W_{0}-W_{\mathrm{F}}\right)}{\partial q_{a}^{\mu}}\right) \\
=H_{0 a}-\frac{1}{2 m}\left(p_{a \mu}-\frac{\partial W_{0}}{\partial q_{a}^{\mu}}\right) \frac{\partial\left(W_{0}-W_{\mathrm{F}}\right)}{\partial q_{a \mu}}
\end{gathered}
$$

have the correct restriction

$$
\begin{aligned}
& \left.H_{\mathrm{F} a}\right|_{\Sigma_{0}}=\left.H_{0 a}\right|_{\Sigma_{0}} \\
& =\frac{1}{2 m} \frac{\partial W_{0}}{\partial q_{a}^{\mu}} \frac{\partial W_{0}}{\partial q_{a}^{\mu}}-\frac{1}{2 m} \frac{\square_{a} R_{0}}{R_{0}}=\frac{1}{2} m,
\end{aligned}
$$

indicating that $W_{0}$ is a solution of the Hamilton-Jacobi equation associated to (31). The true quantum potentials, extended to the entire phase space in a non-trivial way will now depend on the canonical momenta following the relations

$$
\begin{aligned}
V_{\mathrm{F} a} & =\frac{1}{2 m} \frac{\partial\left(W_{0}-W_{\mathrm{F}}\right)}{\partial q_{a}^{\mu}} \frac{\partial\left(W_{0}-W_{\mathrm{F}}\right)}{\partial q_{a \mu}} \\
& -\frac{1}{m} p_{a}^{\mu} \frac{\partial\left(W_{0}-W_{\mathrm{F}}\right)}{\partial q_{a}^{\mu}} \\
& =-\frac{1}{2 m} \frac{\square_{a} R_{0}}{R_{0}}-\frac{1}{m}\left(p_{a}^{\mu}-\frac{\partial W_{0}}{\partial q_{a}^{\mu}}\right) \frac{\partial\left(W_{0}-W_{\mathrm{F}}\right)}{\partial q_{a \mu}},
\end{aligned}
$$

so that its restriction on $\Sigma_{0}$ is the old well-known quantum potential (21):

$\left.V_{\mathrm{Fa}}\right|_{\Sigma_{0}}=V_{0 a}=-\frac{1}{2 m} \frac{\square_{a} R_{0}}{R_{0}}$.

To completely show that $H_{\mathrm{F} a}$ are hamiltonians describing suitable classical analogs of the quantum system in the state $\psi_{0}$, we remark that, from (27) and (29) we have

$u_{a}^{\mu}=\frac{\partial q_{a}^{\mu}}{\partial \tau_{a}}=\frac{1}{m}\left(p_{a}^{\mu}-\frac{\partial\left(W_{0}-W_{\mathrm{F}}\right)}{\partial q_{a \mu}}\right)$,

and hence

$\left.u_{a}^{\mu}\right|_{\Sigma_{0}}=\frac{1}{m} \frac{\partial W_{\mathrm{F}}}{\partial q_{a \mu}}$,

which are always time-like vectors, since ( 30 ) holds. Moreover, since $u_{a}^{\mu}$ are constant vectors, as (28) shows, $u_{a}^{\mu}$ are time-like vectors along all their evolution, so that no causal paradoxes are possible in 
this scheme [8]. However, while we have rectilinear trajectories because $u_{a}^{\mu}$ are constants, velocities and momenta are no longer parallel, so that $p_{a}^{k}$ are not constants. That is why we did not take constrained momenta in our classical formulation: even if $u_{a}^{\mu} u_{a \mu}=1$, as required when $q_{a}^{\mu}$ are true positions and $\tau_{a}$ usual proper times, $p_{a}^{\mu} p_{a \mu}$ is not in general a constant. By summarizing, the action of a quantum potential can be described by means of a sort of gauge field which will not disturb the free motions in the configuration space, even if it deeply influences the canonical momenta.

Of course $H_{\mathrm{Fa}}$ is no unique possible classical ana$\log$ for our quantum system: other extensions to the phase space perhaps exist, as indicated in a pioneering work about this subject [9]. The main interest of the proposition (31) lies in the fact that it shows that for a quantum relativistic system of two particles, a classical analog, satisfying the compatibility conditions (10) and leading to time-like trajectories in the spacetime, always exists. That this result was not at all trivial can be understood from the remark that the usual phase space extension (18) was not a good choice. It should be remarked, however, that this departure from the extension (18), that is compulsory in the relativistic domain, should be enlarged even to the non-relativistic limit. Indeed it makes no sense to adopt two definitely different phase space extension procedures for the relativistic and the nonrelativistic case. Whether this will imply deep modifications even in the usual and well-established causal interpretation of the non-relativistic quantum mechanics will be discussed in forthcoming papers.

Two other topics seem to be worth further developments: first of all our classical analog (31) is still largely undetermined because $W_{\mathrm{F}}$, which is essential in describing the true motion in the spacetime, is not exactly specified. It must only satisfy eq. (30) which is the relativistic analog of the well-known straight light rays equation. However, we will indicate here, as a possibility, the fact that (30) can be interpreted as the real part of the quantum potential type of the non-linear, relativistic Schrödinger equation [10], but we will postpone any deeper examination of this connection to a future discussion. Finally we must remark that the particular relevance of the phase space surface $\Sigma_{0}$ is ill understood from a purely classical standpoint. We can only say here that $\Sigma_{0}$ is determined by means of the function $W_{0}$, which plays a special role because it is the unique solution of the Hamilton-Jacobi equation of the classical analog, which is also a solution of the continuity equation (16). We did not examine this equation in this paper, but it is easy to see that (16) is directly connected with a problem that we have not even mentioned at this stage: namely that of the statistical statements and of the probabilistic interpretation of the theory. Of course that will constitute the second half of every complete reinterpretation of the quantum mechanics.

The author wants to thank Professor Ph. DrozVincent, Professor A. Kyprianidis and Professor J.P. Vigier for substantial discussions and suggestions. He wants to thank also the Italian MPI and INFN for grants that made this research possible.

\section{References}

[1] D. Bohm, Phys. Rev. 85 (1952) 166, 180.

[2] Ph. Droz-Vincent, Rep. Math. Phys. 8 (1975) 79; Ann. Inst. H. Poincaré 27 (1977) 407.

[3] H. Goldstein, Classical mechanics (Addison-Wesley, Reading, 1956);

R. Courant and D. Hilbert, Methods of mathematical physics (Interscience, New York, 1962).

[4] D.G. Currie, T.F. Jordan and E.C.G. Sudarshan, Rev. Mod. Phys. 35 (1963) 350.

[5] Y. Choquet-Bruhat, Géométrie différentielle et systèmes extérieurs (Dunod, Paris, 1968).

[6] N. Cufaro Petroni, Ph. Droz-Vincent and J.P. Vigier, Lett. Nuovo Cimento 31 (1981) 415.

[7] A. Kyprianidis, Phys. Lett. A 111 (1985) 111

[8] N. Cufaro Petroni, in: Open questions in quantum physics, eds. G. Tarozzi and A, van der Merwe (Reidel, Dordrecht, 1985).

[9] Ph. Droz-Vincent, Lett. Nuovo Cimento 40 (1984) 165.

[10] R.W. Hasse, Z. Phys. B 37 (1980) 83; F. Guerra and M. Pusterla, Lett. Nuovo Cimento 34 (1982) 351. 